\title{
Relaxation of Electronic Angular Momentum in Kramers Systems with Strong Spin-Orbit Coupling. 1. Atomic Radicals in Solution*
}

\author{
By Yuri A. Serebrennikov and Ulrich E. Steiner \\ Fakultät für Chemie, Universität Konstanz, D-78434 Konstanz, Germany
}

\section{Spin-orbit coupling / Relaxation / Kramers systems / Atomic radicals}

A theoretical treatment of electron angular momentum relaxation in atoms induced by electric field fluctuations in a liquid solvent cage is presented. It is shown that in the case of weak interaction between solvent shell and a "target" atom the rate of transitions between the components of a Kramers doublet decreases sharply with the increase of adiabatic splitting between the terms of atomic fine-structure. An analytical expression for the rate of solvent induced angular momentum relaxation in the ground state of halogen atoms is obtained. This process is shown to be independent of the strength of spin-orbit coupling in the system.

Der Artikel gibt eine theoretische Behandlung der Relaxation des elektronischen Drehimpulses von Atomen in flüssiger Lösung auf Grund von Fluktuationen des elektrischen Feldes im Lösungsmittelkäfig. Es wird gezeigt, daB im Falle schwacher Wechselwirkung zwischen Solvatsphäre und dem solvatisierten Atom die Übergangsraten zwischen den Komponenten eines Kramers-Dubletts mit zunehmender adiabatischer Aufspaltung zwischen den atomaren Feinstrukturtermen sehr stark abnimmt. Für die Ratenkonstante der Lösungsmittel-induzierten Relaxation des elektronischen Drehimpulses von Halogenatomen wird ein analytischer Ausdruck abgeleitet. Das Ergebnis zeigt, daß hier die Relaxationsrate unabhängig ist von der Stärke der Spin-Bahn-Kopplung.

\section{Introduction}

The theory of spin-lattice relaxation (SLR) of systems with completely quenched orbital momentum in magnetically dilute liquids is well de-

* Presented at the International Symposium "Magnetic Field and Spin Effects in Chemistry and Related Phenomena", 26-31 July 1992. Konstanz, Germany. 
veloped. Detailed treatments may be found in many monographs (see e.g. $[1,2])$. It is weil known that in free radicals and transition metal complexes with an electron spin $S=1 / 2$ and nondegenerate orbital ground state the SLR process is induced by rotational modulation of (i) anisotropic Zeeman interaction, (ii) spin-rotational coupling, and (iii) anisotropic hyperfine interaction. It is important to note that in the former two cases spinorbit coupling (SOC) creates the fundamental connection between the spin subsystem and the bath, and thus, is responsible for SLR.

Electron spin may also be relaxed by processes not related to rotational motion. The thermal molecular vibrations and/or collisions with surrounding diamagnetic particles lead to a random modulation of the interatomic electric field potential, which, through SOC, affects the spin and induces the SLR process. This type of mechanism, first considered for crystals by Kronig [3] and van Vleck [4] has been adapted to liquids by Altshuler and Valiev [5] and, in more detail, by Kivelson [6].

Both molecular rotations and electric field fluctuations lead to the following type of expression for the rate constant of SLR

$$
1 / T_{1} \propto(\lambda / \Delta)^{2},
$$

which applies in the limit

$$
(\Delta / \hat{\lambda})^{2} \gg 1 \text {. }
$$

Here $\lambda$ is an effective SOC constant and $\Delta$ is the basic energy gap ("chemical splitting") between electronic states of different orbital symmetry $[1,2]$.

The direct extrapolation of the functional dependence (1) to systems with near degenerate orbital ground states and large $\lambda$ (i.e. $10^{2}-10^{3} \mathrm{~cm}^{-1}$ ) which would lead to extremely short SLR times, is invalid since it conflicts with condition (2). There is special interest, however, in electron SLR under conditions of such strong SOC. Here we specially mention NMR spectroscopy of paramagnetic complexes $[7,8]$ from which valuable information on structural and dynamic molecular properties can be obtained and magnetic field and spin effects in certain chemical reactions [9-11]. It is essential to this "spin chemistry" that in intermediates such as radical pairs, diradicals, and triplet exciplexes electron spin controls chemical reactivity and any type of spin motion. including spin relaxation. may become a rate-determining step in the process of chemical transformation if the chemical lifetime is comparable to the characteristic time of the spin process.

So far, most studies of spin chemical effects have focussed on radical pair processes in the ns-time-scale. However, the rapid development of ultrafast spectroscopy and its application to studies of geminate atomic recombination in liquid phase have revealed the importance of electron (spin and orbital) angular momentum relaxation in ps processes. For example, it has been shown that unquenched orbital momentum and strong SOC in photolytically generated pairs of $\mathrm{Br}$ and $\mathrm{I}$ atomic radicals are 
responsible for the breakdown of the simple diffusion model of the "cage effect" in these reactions [12]. Recombination of these atomic radical pairs is controlled by the total angular momentum quantum number which may change during a diffusion separation as a consequence of individual atomic angular momentum relaxation at distances where the exchange coupling between the two angular momenta has become negligible [13]. The situation here is quite analogous to that of the well known radical pair mechanism where individual radical spin relaxation (in addition to coherent spin motion driven e.g. by isotropic hyperfine interaction) causes $S / T$ transitions of the pair spin and thereby alters the pair reactivity in a subsequent reencounter [9-11].

An important role of unquenched orbital momentum has also been demonstrated in electron transfer reactions between triplet excited organic molecules and inorganic anions [14-16]. It was pointed out that the quantum yield of free radical formation reflects the degree of "intra radical SOC" (IRSOC [15]) that is particularly pronounced in linear radicals (e.g. it is apparent in the linear $\mathrm{N}_{3}$ radical but not in the bent $\mathrm{NO}_{2}$ radical) and in atomic radicals $\left(\mathrm{I}^{\circ}, \mathrm{Br}^{\circ}, \mathrm{Cl}^{\circ}\right)$.

These examples may suffice to indicate that the problem of electronic angular momentum relaxation in liquid phase for Kramers systems with strong SOC and unquenched orbital momentum is not of purely theoretical interest. It should be stressed, that when SOC is so strong that inequality (2) and Eq. (1) which is based on it, do not hold, one may expect a qualitatively different type of relaxation.

The problem of angular momentum relaxation in atoms through the effect of electric field perturbations exhibited in binary collisions with diamagnetic collision partners in the gas phase has been dealt with in some detail in the literature. The basic theoretical approach is due to Gordeev et al. [17]. Sceats [13] adopted the gas-phase model of collision induced adiabatic relaxation of $J=3 / 2$ angular momentum to calculate solvent induced angular momentum relaxation of iodine atoms with regard to its effect on pair recombination probability in the liquid cage. It may be questionable. how far the binary collision model suitable for the gas phase can be adapted to achieve a realistic description of the situation in liquid solution, where the "target" particle (i.e. an atom or molecule with non-zero spin and orbital angular momentum) is irregularly surrounded by many nearest neighbours creating a fluctuating anisotropic electric field perturbing the electronic orbitals of the particle. In the present work we want to present the basic elements of a treatment of electron angular momentum relaxation in liquids, whereby we adopt the latter point of view in describing the effect of the surrounding solvent shell.

While the final goal of our studies is the understanding of electron angular momentum relaxation in molecules and transition metal complexes with near orbital degeneracy, we will concentrate here on the consideration 
of dilute liquid solutions of atomic radicals, representing the simplest systems which clearly exhibit the basic peculiarities of relaxation in systems with orbitally degenerate electronic ground states. We believe that the theoretical study presented here is a useful step towards developing the adequate theoretical basis for treating the more complicated situations encountered in reaction kinetic spin-orbit effects as mentioned above.

\section{Theory}

As a simplest example we shall consider the case of an atom with a single electron $(\lambda>0)$ or hole $(\lambda<0)$ in the $p$-shell. Under the action of SOC

$$
\mathrm{H}_{0}=\lambda \mathbf{L} \cdot \mathbf{S}
$$

the $(2 L+1)(2 S+1)$ manifold of states splits into a series of levels characterized by the total angular momentum. For our example $L=1$ and $S=$ $1 / 2$. Thus two energy levels are obtained corresponding to $J$ quantum numbers $1 / 2$ and $3 / 2$. The neighbouring particles produce an anisotropic electric field potential $V$ in the region of the atom, which can be expressed as a sum of the form

$$
V=\sum b_{k \mathrm{q}} Y_{k \mathrm{q}} \text {. }
$$

Here $b_{k \mathrm{q}}$ and $Y_{k \mathrm{q}}$ have their usual meanings [2]. For Kramers systems with strong SOC $(\lambda \gg \Delta)$ the effect of this weak perturbation ("ligand field") is partially to lift the $(2 J+1)$ degeneracy of each level of the spin-orbit multiplet of given $J>1 / 2$.

All matrix elements of $V$ are between atomic states of $L=1$. Thus, only terms with $k=2$ in the expansion of the potential (Eq. 4) give a nonvanishing result [2]. The action of $V$ in the space of $p$-functions may be readily expressed in terms of unit irreducible tensor operators $T_{k \mathrm{q}}(L=1)$ determined in the laboratory $(L)$ frame [18]:

$$
V(\Omega)=\sum(-)^{\mathbf{q}} T_{2-\mathrm{q}} \chi_{2 \mathrm{q}}(\Omega),
$$

where

$$
\chi_{2 \mathbf{q}}(\Omega)=\sqrt{2 / 3} \Delta \mathscr{D}_{0 \mathbf{q}}^{2}(\Omega)+\varepsilon\left[\mathscr{D}_{2 \mathbf{q}}^{2}(\Omega)+\mathscr{D}_{2 \mathbf{q}}^{2}(\Omega)\right]
$$

involve only "ligand field" variables. Here $\mathscr{D}_{p q}^{2}(\Omega)$ is the corresponding Wigner rotational matrix [18], the set of the Euler angles $\Omega$ represents the instantaneous orientation of $L$-frame in the coordinate system defined by the main axes $(i=x, y, z)$ of the electric field tensor $V_{i i}$; parameters $\Delta$ and $\varepsilon$ define the electric field splitting of $p$-orbitals in the absence of SOC:

$$
\Delta=-3 V_{z z} / 2 ; \varepsilon=\left(V_{y y}-V_{x x}\right) / 2 \text {. }
$$


Accordingly, the dynamical evolution of the system is governed by the following Hamiltonian:

$$
\mathrm{H}=\mathrm{H}_{0}+V(\Omega) \text {. }
$$

An anisotropic "ligand field" potential destroys the spherical symmetry of the system. As a result, the orbital angular momentum is no longer a constant of motion. In other words, $\mathbf{L}^{2}$ does not commute with $V$ and for strong enough fields $\left(\Delta, \varepsilon \gg k_{B} T, \lambda\right)$ the expectation values of $\mathbf{L}$-components equal zero. This situation corresponds to polyatomic molecules and complexes of low symmetry, where the orbital angular momentum is quenched. In a strong axially symmetric electric field $\left(\varepsilon=0, \Delta \gg k_{B} T\right)$ the operator $L_{z}$ does commute with $V(\Omega=0)$ and its expectation value is a conserved quantity. This situation corresponds, for instance, to a nonrotating diatomic molecule. In such a case the motion of the electron may be described as a rotation about the axis $z$ of axial symmetry. This orbital current produces a magnetic field, which is parallel to the molecular axis and interacts with the spin. Thus, due to SOC, the spin is effectively coupled with the anisotropic part of the liquid cage potential $V$ ("ligand field" in a general sense).

In liquids, the interaction of a "target" particle with near neighbours fluctuates in time. This process induces relaxation of orbital angular momentum and hence of spin. The motion of either surrounding molecules, or the "target" particle itself leads to a stochastic variation of $V(\Omega)$. The basic problem is the calculation of an average response of the system to this random perturbation. In the case under consideration $V$ depends on two multidimensional stochastic variables: $\Omega$ and $\mathbf{v}=\{\Delta, \varepsilon\}$. Since the former is determined by a variation of the electric field tensor in the angular space and the latter by a variation of the distance between the atom and its environment, they are set to vary independently.

If the orientation of the main axes of the electric field tensor is fixed in time ( $\Omega=$ constant), the temporary fluctuations of $\mathbf{v}$ could independently induce relaxation of electron angular momentum. This limit corresponds to the action of "stretching" modes of the collective motion and is related to McConnell's "spin-orbit pulse" mechanism of SLR [19]. The "librational" modes, which only change the orientation $\Omega$ lead to the "rotational spinorbit" type relaxation mechanisms (see e.g. [20] and Refs. therein).

However, as was already mentioned in the Introduction, almost all of the studies concerned with the problem of SLR in liquid solutions were restricted by the limit of weak effective $\operatorname{SOC}(\lambda \ll \Delta)$ in the system. When inequality (2) inverts sign the spin and orbital variables are strongly coupled and the equation of motion of the full $(L, S)$-system must be solved before the calculation of any macroscopic observable.

Thus, to find the autocorrelation function of $\mathbf{J}$ : 


$$
K_{\mathbf{J}}(t)=\operatorname{Tr}\left\{\varrho^{\text {eq }} \mathbf{J}^{\dagger}\langle\mathbf{J}(t)\rangle\right\},
$$

we have to average, $\langle\ldots\rangle$, over all possible realizations of the general solution of the Liouville equation

$$
(d / d t) \mathbf{J}(t)=i \mathrm{H}^{x}(t) \mathbf{J}(t)
$$

describing the dynamical motion of the electronic angular momentum of a "target" atom along any stochastic trajectory $V(t)$. In Eqs. (9) and $(10) \varrho^{\text {eq }}$ is the equilibrium density operator, $\hbar=1, \mathrm{H}^{x} \mathbf{J}=[\mathrm{H}, \mathbf{J}]$.

If the correlation time of $V(\Omega)$ is small in comparison with the characteristic time of the response of the quantum system to the action of $V$, we can use a standard procedure of stochastic perturbative expansion [21] to deduce from Eq. (10) the kinetic equation for the averaged operator $\langle\mathbf{J}(t)\rangle$.

For the first nonvanishing term in the so-called G-ordering representation [21] we have

$$
(d / d t)\langle\mathbf{J}(t)\rangle=-\int_{0}^{t} \hat{M}\left(t-t^{\prime}\right)\left\langle\mathbf{J}\left(t^{\prime}\right)\right\rangle d t^{\prime},
$$

where

$$
\hat{M}(\tau)=\left\langle V^{x}\left(\Omega_{\tau}\right) \exp \left(i \mathrm{H}_{0}^{x} \tau\right) V^{x}(\Omega)\right\rangle .
$$

Note, that due to the assumed isotropy of the liquid $\mathrm{H}_{0}^{x}\langle\mathbf{J}(t)\rangle=0$ and the Wigner-Eckart theorem [18] holds true for the matrix elements of the averaged operator $\langle\mathbf{J}(t)\rangle$ (compare, e.g. Ref. [22]). Solving Eq. (11) with the help of Laplace transformation and substituting the solution into Eq. (9) we obtain

$$
\tilde{K}_{J}(p)=\operatorname{Tr}\left\{e^{\text {eq }} \mathbf{J}^{\dagger} \frac{1}{1 p+\hat{\tilde{M}}(p)} \mathbf{J}\right\},
$$

where

$$
\tilde{K}_{j}(p)=\int_{0}^{\infty} e^{-\mathrm{pt}} K_{\mathrm{J}}(t) d t .
$$

The frequency dependence of the superoperator $M(p)$ reflects the nonMarkovian character of the perturbation theory as presented in Eq. (11) (an open upper limit of time-integration).

It should be noted, that usually SLR has been treated in the Markovian limit [1]. In this approximation the relaxation superoperator in Eq. (13) does not depend on frequency and contains no information about the evolution of the system over times comparable with and less than the correlation time of the perturbation. This approach to the problem is well satisfied when the time independent terms in the Hamiltonian are of the 
order of magnetic dipole-dipole or rotational energy so that the perturbation of internal motion of the system has nonadiabatic character. However, this is not the case when dealing with a system with large energy gap between terms, i.e. if the frequency of internal motions is determined by $\left\|\mathrm{H}_{0}\right\| \approx \lambda=10^{2}-10^{3} \mathrm{~cm}^{-1}$. We shall return to this point below.

To proceed further it is convenient to choose the following set of irreducible tensor operators as a basis in the Liouville space

$$
\left.T_{x Q}(J J)=\sum_{\mu \mu \prime}(-)^{J-\mu} C_{J \mu \prime J-\mu}^{H Q}\left|J \mu^{\prime}\right\rangle\left\langle J \mu|\equiv| T_{x Q}(J J)\right\rangle\right\rangle
$$

where

$$
|J \mu\rangle=\sum_{m m^{\prime}} C_{L m S m^{\prime}}^{J \mu}|L m\rangle\left|S m^{\prime}\right\rangle
$$

is the general wave function of the compound $(L, S)$-system, $C . .$. is the Clebsch-Gordan coefficient [18]. In this representation we obtain, after summation over magnetic quantum numbers,

$$
\begin{aligned}
& K_{J}(p)=1 / 3 \sum_{Q J J_{1}} \varrho_{J}^{\mathrm{eq}}\left[J J_{1}(J+1)\left(J_{1}+1\right)(2 J+1)\left(2 J_{1}+1\right)\right]^{1 / 2} \\
& \times\left\langle\left\langle T_{1 Q}(J J)\left|\frac{1}{\hat{1} p+\hat{\tilde{M}}(p)}\right| T_{1 Q}\left(J_{1} J_{1}\right)\right\rangle\right\rangle .
\end{aligned}
$$

Here

$$
\begin{aligned}
& \left\langle\left\langle T_{1 Q}(J J)|\hat{\tilde{M}}(p)| T_{1 Q}\left(J_{1} J_{1}\right)\right\rangle\right\rangle \equiv \hat{\tilde{M}}_{J J_{1}}(p) \\
& =4 / 3\left\langle\Delta^{2}\right\rangle \sum_{J_{2}}\left[\delta_{J_{J_{2}}}+(-)^{J+\mathrm{J}_{1}}(2 J+1)\left\{\begin{array}{lll}
J_{J_{1}} & J_{1} & 2
\end{array}\right\} \delta_{J_{1} J_{2}}\right] \\
& \times\left(2 J_{2}+1\right)\left\{\begin{array}{lll}
J_{L} J_{L} & 2 \\
L
\end{array}\right\}^{2} \operatorname{Re} \widetilde{F}_{2}\left(p+i \omega_{J J_{2}}\right),
\end{aligned}
$$

$\{\cdots\}$ is the $6 j$ symbol [18],

$$
\omega_{J J_{2}}=(\lambda / 2)\left[J(J+1)-J_{2}\left(J_{2}+1\right)\right] .
$$

$\tilde{F}_{2}$ is the spectral density of the normalized to unity autocorrelation function of the electric field potential:

$$
F_{2}(t)=\langle\Delta(t) \Delta(0)\rangle\left\langle\mathscr{D}_{00}^{2}\left(\Omega_{t}\right) \mathscr{D}_{00}^{2}(\Omega)\right\rangle /\left\langle\Delta^{2}\right\rangle .
$$

Two simplifications were made in deriving Eq. (16): it is assumed that (i) $\varepsilon=0$, i.e. the "ligand field" potential has axial symmetry; (ii) the relaxation time $\tau_{2} \equiv \tilde{F}_{2}(p=0)$ is small in comparison with the inverse of the amplitude of perturbation

$$
\left\langle\Delta^{2}\right\rangle \tau_{2}^{2} \ll 1 \text {. }
$$


The nondiagonal elements of the relaxation matrix Eq. (17) are responsible for the transitions between the different levels of the atomic fine structure. On the other hand, relaxation inside the $J$-multiplets is governed by the diagonal matrix elements of $\tilde{M}(p)$.

It is important to note, that the properties of $6 j$-symbols in Eq. (17) forbid transitions inside the $J=1 / 2$ doublet. It is the result of a fundamental symmetry restriction: an electric field cannot split or induce transitions between the components of a Kramers doublet. Therefore, if the ground state of a spin-orbit multiplet corresponds to $J=1 / 2(\lambda>0)$, the relaxation of the electronic angular momentum may occur only by transitions into the upper $(J=3 / 2)$ multiplet. This process is the direct analogue of the Orbach mechanism of phonon induced SLR [23]. On the other hand, if the system under study has a $J>1 / 2$ ground state $(\lambda<0)$, the fluctuating "ligand field" may split the levels of the ground multiplet and induce relaxation between states differing only in $J$-projection. Clearly this process is the direct analog of so-called " $m$-diffusion" of rotational angular momentum [24].

The value which is of main interest to us is the relaxation time of $J$ :

$$
\begin{aligned}
T_{J}= & \tilde{K}_{J}(p=0) / K_{J}(t=0)=K_{J}^{-1}(t=0) \times \\
& \times \sum_{J J_{1}} \varrho_{J}^{\mathrm{eq}}\left[J J_{1}(J+1)\left(J_{1}+1\right)(2 J+1)\left(2 J_{1}+1\right)\right]^{1 / 2} \hat{\tilde{M}}_{J J_{1}}^{-1}(p=0) .
\end{aligned}
$$

For halogen atoms (ground state ${ }^{2} P_{3 / 2}, L=1, S=1 / 2, \lambda<0$ ) the matrix of the relaxation operator Eq. (17) takes the form

$$
\begin{gathered}
M_{J J}(p=0)=4 / 9\left\langle\Delta^{2}\right\rangle \\
\times\left[\begin{array}{cc}
(2 / 5) \tau_{2}+1 / 2 \operatorname{Re} \tilde{F}_{2}\left(i \omega_{0}\right) & 1 / \overline{10} \operatorname{Re} \tilde{F}_{2}\left(i \omega_{0}\right) \\
1 / \sqrt{10} \operatorname{Re} \tilde{F}_{2}\left(-i \omega_{0}\right) & \operatorname{Re} \tilde{F}_{2}\left(-i \omega_{0}\right)
\end{array}\right] \begin{array}{l}
(J=3 / 2) \\
(J=1 / 2)
\end{array}
\end{gathered}
$$

where $\omega_{0}=3|\lambda| / 2$.

Thus, the final result depends on the explicit form of the autocorrelation function $F_{2}(t)$. If the process of electric field fluctuations is treated as an external random noise (semiclassical approximation, bath at infinite temperature) then

$$
\operatorname{Re} \tilde{F}_{2}\left(i \omega_{0}\right)=\operatorname{Re} \tilde{F}_{2}\left(-i \omega_{0}\right) .
$$

If, following Kivelson [6], in addition we assume an exponential law for the relaxation of the electric field potential (Markovian limit): $F_{2}(t)=$ $\exp \left(-t / \tau_{2}\right)$, then

$$
\operatorname{Re} \tilde{F}_{2}\left( \pm i \omega_{0}\right)=\tau_{2} /\left[1+\left(\omega_{0} \tau_{2}\right)^{2}\right] \text {. }
$$

It is easy to see from Eqs. (22) - (24) that within the framework of these model approximations we obtain: (i) equal "up" $(J=3 / 2 \rightarrow J=1 / 2)$ and "down" $(J=1 / 2 \rightarrow J=3 / 2)$ transition rates, leading to an equal population of $J$-levels; (ii) quadratic decrease of the probability of these transitions with the growth of $\omega_{0}$, provided $\omega_{0} \tau_{2} \gg 1$. However. both of these predictions are 
quite unacceptable in the case under consideration since in halogen atoms the energy gap between the fine-structure terms significantly exceeds $k_{B} T$ in a room temperature liquid. In such a case one must expect an exponential decrease of the rate of transitions between the widely separated electronic terms $\left(\omega_{0} \gg k_{B} T\right)$ with the growth $\omega_{0}$, or in other words, an effective adiabatic isolation of $J=3 / 2$ and $J=1 / 2$ multiplets.

Indeed, if we consider, for example, the function [25]

$$
F_{2}(t)=\left(2 \tau_{2} / \pi\right)^{2}\left[t^{2}+\left(2 \tau_{2} / \pi\right)^{2}\right]^{-1},
$$

then we have

$$
\operatorname{Re} \widetilde{F}_{2}\left( \pm i \omega_{0}\right)=\tau_{2} \exp \left[-2 \omega_{0} \tau_{2} / \pi\right] \text {. }
$$

Thus, non-Markovian stochastic theory even in a semiclassical approximation predicts the correct functional dependence of the spectral density of electric field fluctuations and hence the probability of corresponding transitions upon the level spacing in a quantum subsystem. However, in any "classical" stochastic model $(\hbar=0)$ the detailed balance condition

$$
\operatorname{Re} \widetilde{F}_{2}\left(i \omega_{0}\right)=\exp \left(-h \omega_{0} / k_{B} T\right) \operatorname{Re} \widetilde{F}_{2}\left(-i \omega_{0}\right)
$$

is not satisfied.

However, to calculate $T_{J}$ in the halogen atoms it is not necessary to know the explicit form of quantal corrections to the relaxation matrix Eq. (22). In such a case, $\left(\omega_{0} \gg k_{B} T\right.$ and $\left.1 / \tau_{2}\right)$, transitions between widely spaced levels $J=3 / 2$ and $J=1 / 2$ become hardly probable, $\tilde{M}_{J J_{1}}(p=0)=\tilde{\bar{M}}_{J J}$ $(p=0) \delta_{J J_{1}}$. Electronic angular momentum may relax only due to transitions inside the corresponding spin-orbit multiplet (" $m$-diffusion"). Thus. from Eqs. (21) and (22) we obtain the following final result

$$
1 / T_{\mathrm{J}=3 / 2}=8 / 45\left\langle\Delta^{2}\right\rangle \tau_{2} \text {. }
$$

As was already mentioned, in conformity with Kramers theorem an electric field cannot split and induce transitions between the components $m=1 / 2$ and $m=-1 / 2$ of a Kramers doublet. On the other hand, the probability of transitions into the levels of the upper multiplet, which may lead to a relaxation of an effective spin $=1 / 2$ is exponentially small in the adiabatic limit $\left(\omega_{0} \gg 1 / \tau_{2}, k_{B} T\right)$. Thus, in the case of weak action [cf. Eq. (20)] of the fluctuating electric field an increase of SOC in systems with $J=$ $1 / 2$ ground state will lead to a strong hindering of the relaxation process!

Although the result [Eq. (28)] depends upon the parameter $\left\langle\Delta^{2}\right\rangle$, which is difficult to evaluate, it provides a clear qualitative picture of the process and may be used to obtain reasonably reliable estimates of the magnitude of the effect. In liquids the stochastic variation of the interparticle potential has essentially a multibody character and depends largely upon the rather fast translational fluctuations $\tau_{2} \approx 10^{-12}-10^{-13} \mathrm{~s}$. We may therefore assume that for liquid solutions of uncharged atomic radicals inequality (20) holds. Combining it with Eq. (28) it is easy to see that in liquid solutions 
of halogen atoms $1 / T_{\mathrm{J}} \lesssim 10^{11} \mathrm{~s}^{-1}$ and does not depend upon SOC and the strength of external magnetic field (up to $10 T$ ). This fast relaxation may result in an effective spin equilibration and quenching of MFEs in reactions of halogen atomic radicals [26]. Since the relaxation rate is proportional to $\left\langle\Delta^{2}\right\rangle \tau_{2}$ one can predict a lowering of $1 / T_{\mathbf{J}}$ in more inert solvents, or at higher temperatures.

\section{Conclusions}

The theoretical study presented here clearly reflects the following qualitative picture: in systems with orbital degeneracy the strong SOC $\left(\lambda^{2} \gg\left\langle\Delta^{2}\right\rangle\right)$ defines the quasi stationary electronic states and the relaxation comes from the stochastic time dependence of electric field potential. As a result, when the action of this potential is low enough, $\left\langle\Delta^{2}\right\rangle \tau_{2}^{2} \ll 1$, the relaxation rate is proportional to $\left\langle\Delta^{2}\right\rangle$ the mean square of the electric field perturbation. Moreover, for systems with $J>1 / 2$ ground state $1 / T_{\mathfrak{J}}$ does not depend on the strength of SOC, since relaxation is mainly due to transitions within the same $J$-multiplet. On the contrary, the relaxation inside a Kramers doublet $(J=1 / 2)$ may occur only by transitions into the levels of the upper spin-orbit multiplet.

It is well known, that under adiabatic conditions $\left(\lambda \gg 1 / \tau_{2}, k_{B} T\right)$ nonMarkovian stochastic perturbation theory, which leads to the general expression for $T_{J}$ (Eq. 21), predicts an exponential decrease of the probability of transitions between electronic terms with increasing energy gap between them. In other words when $\lambda \tau_{2} \gg 1$, the system remains in the ground term for almost infinite time. Thus, as follows from Eqs. (21), (22) and (26), in the case of weak action of the fluctuating electric field potential increasing SOC in systems with formal spin $=1 / 2$ leads to a strong hindering of the relaxation process, provided $\left.\lambda\rangle 1 / \tau_{2}, k_{B} T\right\rangle\left\langle\Delta^{2}\right\rangle^{1 / 2}$.

Thus the theoretical analysis of the limiting case studied in this work, which is opposite to the case of inequality (2), leads to a qualitatively different dependence of $1 / T_{\mathrm{J}}$ upon the strength of SOC than the one predicted by Eq. (1). At present we have been concerned with liquid solutions of atomic radicals only, i.e. obviously, the simplest systems allowing one to treat the action of the electric field potential as a weak perturbation, and to come to first physically sound results. Naturally, the most interesting case is a strong, slowly fluctuating field $\left.\left.\left(\left\langle\Delta^{2}\right\rangle^{1 / 2}\right\rangle \lambda\right\rangle k_{B} T, 1 / \tau_{2}\right)-\mathrm{a}$ situation encountered in the problem of electronic angular momentum relaxation in linear radicals and paramagnetic complexes of high symmetry. The formulation of the corresponding theory is now in progress.

\section{Acknowledgement}

We are grateful to the Alexander-von-Humboldt foundation for granting a fellowship to Yu. A. S. 


\section{References}

1. A. Abragam, Principles of Nuclear Magnetism. Oxford Univ. Press (1961).

2. A. Abragam and B. Bleany, Electron Paramagnetic Resonance of Transition Ions in Crystals. Academic Press, N. Y. (1970).

3. R. de L. Kronig, Physica 6 (1939) 33.

4. J. Van Vleck, Phys. Rev. 57 (1940) 426

5. S. A. Altshuler and K. A. Valiev, Sov. Phys. JETP 8 (1959) 661.

6. D. Kivelson, J. Chem. Phys. 45 (1966) 1324.

7. J. Kovalevsky, L. Nordenskiöld, N. Benetis and P.-O. Westlund, Prog. Nucl. Magn. Reson. Spectrosc. 17 (1985) 141.

8. L. Banci, I. Bertini and C. Luchinat, Nuclear and Electron Relaxation, Verlag Chemie, Weinheim, 1991.

9. U. E. Steiner and T. Ulrich, Chem. Rev. 89 (1989) 51.

10. U. E. Steiner and H.-J. Wolff, in Photochemistry and Photophysics, J. Rabek, G. Scott, eds., 4 (1991) 1.

11. I. V. Khudyakov, Yu. A. Serebrennikov and N. J. Turro, Chem. Rev. 93 (1993) 537.

12. A. Harris, J. Brown and C. Harris, Ann. Rev. Phys. Chem. 39 (1988) 341.

13. M. Sceats, Chem. Phys. 96 (1985) 299.

14. G. Winter, H. Shioyama and U. E. Steiner, Chem. Phys. Lett. 81 (1981) 547.

15. A. Treinin, I. Loeff, J. K. Hurley and H. Linschitz, Chem. Phys. Lett. 95 (1983) 333.

16. J. K. Hurley, H. Linschitz and A. Treinin, J. Phys. Chem. 92 (1988) 5151.

17. E. Gordeev, E. E. Nikitin and M. Ya. Ovchinnikova, Can. J. Phys. 47 (1969) 1819.

18. D. A. Varshalovich, A. N. Moskalev, and V. K. Khersonsky, Quantum Theory of Angular Momentum, World Scientific, Singapore 1989.

19. H. McConnell, J. Chem. Phys. 34 (1961) 13.

20. M. R. Das, S. B. Wagner and J. H. Freed, J. Chem. Phys. 52 (1970) 5404

21. R. Kubo, J. Math. Phys. 4 (1963) 174.

22. S. I. Temkin and B. M. Abdrakhmanov, Phys. Letters A 155 (1991) 43.

23. R. Orbach, Proc. Phys. Soc. (London) A 77 (1961) 821.

24. A. I. Burshtein and S. I. Temkin. Spectroscopy of Molecular Rotations in Liquids and Gases (1982), Nauka, Novosibirsk (in Russ.).

25. A. I. Burshtein, A. V. Storozhev and M. L. Strekalov, Sov. Phys. JETP 62 (1986) 456.

26. E. Broomhead and K. McLauchlan, J. Chem. Soc. Faraday Trans. (2) 74 (1978) 775. 